\title{
DO GENDER AND PERSONALITY TRAITS (BFI-10) INFLUENCE TRUST? A REPLICATION
}

\section{František Sudzina}

\begin{abstract}
Trust as a concept found its way to business literature and it became a widely-used variable. Societal trust is systematically measured since 1960s. Later, it was discovered that two statements, which were used as opposite ends of dichotomous questions, are not truly opposite. The aim of this article is to investigate if gender and personality traits influence rating of these two statement. And if so, if it is possible to account for these factors and to create a robust trust indicator from these two statements after all. Big Five Inventory-10 is used to measure personality traits. Findings are that one measure of trust is significantly influenced by agreeableness, while the other is possibly influenced by neuroticism but the relationship is only borderline significant. With regards to the second goal, it is not possible to create a single trust indicator with reasonable properties even with adding personality traits into the equation. This article is a replication of a previous study. This study uses 1-5 Likert scales while the previous used 1-7 Likert scales, while all the questions/statements stayed the same. The difference is that both measures (not only the first measure) of trust were significantly influenced by agreeableness, while neuroticism had a borderline significant impact on the first, not the second measure of trust.
\end{abstract}

\section{Keywords}

trust, personality traits, gender, empirical research, quantitative methods

\section{Introduction}

Trust is not something solely in the domain of sociology anymore. It became a widely investigated concept in business research in the last two decades. It influences behavior when it comes to barter exchanges (Ostroy and Starr, 1990), off-line (Calvo Porral and Levy-Mangin, 2016) and on-line purchases (Delina and Drab, 2010). It influences organization behavior as well, e.g. the relationship between a superior and subordinated (Krasman, 2014) or knowledge-sharing (Peralta and Saldanha, 2014).

Historically, the most standard question to measure trust is to ask "Generally speaking, do you believe that most people can be trusted, or can't you be too careful in dealing with people?" It was used by Rosenberg (1956) for the first time in the United States. Almond and Verba (1963) used it for the first time on a larger scales in 1960, the study involved five countries. Since then, American National Election Studies included this question in many of its surveys.

According to (Smith, 1997), the order, in which questions are asked, influences answers to the standard question. Respondents tend to opt for the positive answer with a high probability when the standard question is located after several pro-social questions involving e.g. working on community projects or volunteering.

Now that we are aware of the order of questions influencing answers, it is possible to find a more suitable place for the standard question where it is not influenced by previous 
questions. Alternatively, it is possible to use an on-line questionnaire tool and randomize order of the questions - something that was not too realistic 20 years ago.

A more serious problem is that, according to Miller and Mitamura (2003) and Wuthnow (1998), two parts of the standard question, i.e. "most people can be trusted" and "you can't be too careful in dealing with people" are not true opposites but rather two separate questions. When Wuthnow (1998) asked the two questions separately, about one half to two thirds of respondents gave inconsistent answers.

Therefore, the research presented in this article uses the two parts of the standard question as two questions. The aim is to investigate if gender and personality traits influence answers to these two questions. And if so, if it is possible to account for these factors and create a robust single-dimension trust indicator from these two statements after all. It is a replication of previous study (Sudzina, 2016), which used the same statements but with 1-7 Likert scales instead of a 1-5 Likert scales.

The rest of the article is organized in the following way: In the next section, there is a description what data were collected and how, and how they were analyzed. In the following section, results of the analysis are presented. The last section offers conclusions.

\section{Data and methodology}

Data were collected in the spring semester 2014 using a broader on-line questionnaire dealing with personality traits. Respondents were students of Aalborg University. Of 186 students who started, 172 (of whom 106 were male and 66 female) fully filled in the questionnaire.

Trust was measured using the following two statements preceded by question "To what extent do you agree with the following statements?":

- most people can be trusted,

- you can't be too careful in dealing with people

on a 1-5 Likert scale (as opposed to a 1-7 Likert scale in (Sudzina, 2016)) where 1 meant strongly disagrees and 5 stood for strongly agree. For convenience, the former will be addressed in the article as trust and the latter as mistrust (both in italics).

Personality traits were measured using the Big Five Inventory-10, i.e. a 10-item version of the questionnaire for the Big Five Inventory, developed by Rammstedt and John (2007). The instruction was to rate "How well do the following statements describe your personality" with statements "I see myself as someone who..."

... is reserved,

... is generally trusting,

... tends to be lazy,

... is relaxed, handles stress well,

... has few artistic interests,

... is outgoing, sociable,

... tends to find fault with others,

... does a thorough job,

... gets nervous easily,

... has an active imagination

on a 1-5 Likert scale (as opposed to a 1-7 Likert scale in (Sudzina, 2016)) where 1 meant strongly disagrees and 5 stood for strongly agree. Extraversion was calculated as an average of the 1st (reversed-scored) and the 6th answer, agreeableness as an average of the 2nd and 
the 7 th (reversed-scored) answer, conscientiousness as an average of the 3rd (reversed-scored) and the 8th answer, neuroticism as an average of the 4th (reversed-scored) and the 9th answer, and openness to experience as an average of the 5th (reversed-scored) and the 10th answer. Cronbach alphas for personality traits will not be reported since the Big Five Inventory-10 (Rammstedt and John, 2007) was not constructed with this statistics in mind.

This questionnaire was preceded by another questionnaire, approximately a week before, it contained the same Big Five Inventory-10, and respondents were asked to save the answers and provide then again later. So one of the questions not analyzed here is whether the respondents entered their answers from a week before or they filled in their current answers. Of 172 respondents, 63 personality traits ratings were from previous week, and 109 were recent.

The questionnaire contained additional questions which were not used in the analysis presented in this article.

General linear model was used to analyze impact of gender and five personality traits (extraversion, agreeableness, conscientiousness, neuroticism, openness to experience) on trust. Parameter estimates are provided in tables in order to communicate the direction of relationships. A multivariate approach was used. Pearson product-moment correlation coefficient was used to measure correlation. SPSS software was used for the analysis.

\section{Results}

The correlation coefficient between trust and mistrust in the sample at hand is -.226 ( $\mathrm{p}$-value $=.003)$. In (Sudzina, 2016), the correlation coefficient was -.339 (p-value $<.001)$. It confirms what Miller and Mitamura (2003) and Wuthnow (1998) discovered, i.e. that two statements in the standard question for measuring trust are not opposite. If they were opposite, the correlation coefficient would be (close to) -1 .

Parameter estimates of general linear model explaining trust are provided in Table 1. With regards to the explanatory power, $\mathrm{R}^{2}=.066, \mathrm{R}_{\text {adj }}^{2}=.032, \mathrm{p}$-value $=.078$. In (Sudzina, 2016), such model had a higher explanatory power, $\mathrm{R}^{2}=.157, \mathrm{R}_{\text {adj }}^{2}=.138$, $\mathrm{p}$-value $<.001$.

\begin{tabular}{l|l|l|l|l}
\hline Parameter & \multicolumn{1}{|c|}{$\mathrm{B}$} & \multicolumn{1}{|c|}{ Std. Error } & $\mathrm{t}$ & \multicolumn{1}{c}{ Sig. } \\
\hline Intercept & 1.362 & .756 & 1.801 & .073 \\
\hline Extraversion & -.037 & .114 & -.321 & .748 \\
\hline Agreeableness & .310 & .123 & 2.517 & .013 \\
\hline Conscientiousness & .162 & .111 & 1.457 & .147 \\
\hline Neuroticism & .126 & .107 & 1.173 & .242 \\
\hline Openness to experience & .019 & .109 & .178 & .859 \\
\hline [Gender=male] & -.004 & .168 & -.023 & .982 \\
\hline
\end{tabular}

Table 1 Parameter estimates of impact of gender and personality traits on trust

The impact of agreeableness is the only significant, it was also significant and actually the only with a p-value below .1 in (Sudzina, 2016). 
Parameter estimates for the best streamlined model are provided in Table 2. With regards to the explanatory power, $\mathrm{R}^{2}=.045, \mathrm{R}_{\text {adj }}^{2}=.039$, $\mathrm{p}$-value $=.005$. In (Sudzina, 2016), the model containing only agreeableness had a higher explanatory power, $\mathrm{R}^{2}=.133, \mathrm{R}_{\text {adj }}^{2}=.130$, $\mathrm{p}$-value $<.001$.

\begin{tabular}{l|l|l|r|c}
\hline Parameter & \multicolumn{1}{|c|}{$\mathrm{B}$} & \multicolumn{1}{|c|}{ Std. Error } & $\mathrm{t}$ & \multicolumn{1}{c}{ Sig. } \\
\hline Intercept & 2.182 & .387 & 5.633 & .000 \\
\hline Agreeableness & .311 & .110 & 2.829 & .005 \\
\hline
\end{tabular}

\section{Table 2 Parameter estimates of impact of agreeableness on trust}

Parameter estimates for the second best streamlined model are provided in Table 3 . With regards to the explanatory power, $\mathrm{R}^{2}=.022, \mathrm{R}_{\text {adj }}^{2}=.017, \mathrm{p}$-value $=.050$.

\begin{tabular}{l|l|l|l|l}
\hline Parameter & \multicolumn{1}{|c|}{$\mathrm{B}$} & \multicolumn{1}{|c|}{ Std. Error } & $\mathrm{t}$ & \multicolumn{1}{c}{ Sig. } \\
\hline Intercept & 2.548 & .367 & 6.945 & .000 \\
\hline Conscientiousness & .201 & .102 & 1.972 & .050 \\
\hline
\end{tabular}

\section{Table 3 Parameter estimates of impact of conscientiousness on trust}

Parameter estimates of general linear model explaining mistrust are provided in Table 4. The model per se is not significant, $\mathrm{R}^{2}=.047, \mathrm{R}^{2}$ adj $=.012$, $\mathrm{p}$-value $=.234$. In (Sudzina, 2016), such model was significant $\left(\mathrm{R}^{2}=.075, \mathrm{R}_{\text {adj }}^{2}=.055\right.$, $\mathrm{p}$-value $\left.=.001\right)$.

\begin{tabular}{l|l|l|l|l}
\hline Parameter & \multicolumn{1}{|c|}{$\mathrm{B}$} & \multicolumn{1}{|c|}{ Std. Error } & $\mathrm{t}$ & \multicolumn{1}{c}{ Sig. } \\
\hline Intercept & 2.543 & .698 & 3.644 & .000 \\
\hline Extraversion & -.083 & .105 & -.787 & .433 \\
\hline Agreeableness & -.120 & .114 & -1.053 & .294 \\
\hline Conscientiousness & .123 & .102 & 1.200 & .232 \\
\hline Neuroticism & .161 & .099 & 1.620 & .107 \\
\hline Openness to experience & .083 & .100 & .823 & .412 \\
\hline [Gender=male] & .142 & .155 & .918 & .360 \\
\hline
\end{tabular}

Table 4 Parameter estimates of impact of gender and personality traits on mistrust

In (Sudzina, 2016), the impact of agreeableness was significant, and the impact of neuroticism was borderline significant $(\mathrm{p}$-value $=.098$ ). The significance of neuroticism is similar also in this research. 
Parameter estimates for a streamlined model are provided in Table 5. There was no model with $\mathrm{p}$-values below .05 . With regards to the explanatory power, $\mathrm{R}^{2}=.020, \mathrm{R}_{\text {adj }}^{2}=.014$, $\mathrm{p}$-value $=.063$.

\begin{tabular}{l|l|l|l|l}
\hline Parameter & \multicolumn{1}{|c|}{$\mathrm{B}$} & \multicolumn{1}{|c|}{ Std. Error } & $\mathrm{t}$ & \multicolumn{1}{c}{ Sig. } \\
\hline Intercept & 2.625 & .230 & 11.421 & .000 \\
\hline Neuroticism & .160 & .086 & 1.873 & .063 \\
\hline
\end{tabular}

\section{Table 5 Parameter estimates of impact of neuroticism on mistrust}

The second goal of the article was to investigate if it is possible to account for personality traits and to create a robust single-dimension trust indicator from trust and mistrust questions. Parameter estimates of general linear model explaining trust using mistrust, gender and personality traits are provided in Table 6. The explanatory power of the model is higher than of the model without mistrust $\left(\mathrm{R}^{2}=.117, \mathrm{R}^{2}{ }_{\text {adj }}=.080\right.$, $\mathrm{p}$-value $\left.=.004\right)$. In (Sudzina, 2016), such model had a higher explanatory power $\left(\mathrm{R}^{2}=.234, \mathrm{R}_{\text {adj }}^{2}=.215\right.$, $\mathrm{p}$-value $\left.<.001\right)$.

\begin{tabular}{l|l|l|l|l}
\hline Parameter & \multicolumn{1}{|c|}{$\mathrm{B}$} & \multicolumn{1}{|c|}{ Std. Error } & $\mathrm{t}$ & \multicolumn{1}{c}{ Sig. } \\
\hline Intercept & 2.009 & .766 & 2.623 & .010 \\
\hline Mistrust & -.255 & .082 & -3.097 & .002 \\
\hline Extraversion & -.058 & .111 & -.518 & .605 \\
\hline Agreeableness & .280 & .120 & 2.320 & .022 \\
\hline Conscientiousness & .193 & .109 & 1.776 & .078 \\
\hline Neuroticism & .167 & .106 & 1.581 & .116 \\
\hline Openness to experience & .040 & .106 & .381 & .704 \\
\hline [Gender=male] & .032 & .164 & .197 & .844 \\
\hline
\end{tabular}

Table 6 Parameter estimates of impact of mistrust, gender and personality traits on trust

Parameter estimates for a streamlined model are provided in Table 7 . The explanatory power stayed almost the same, $\mathrm{R}^{2}=.115, \mathrm{R}_{\text {adj }}^{2}=.094$, $\mathrm{p}$-value $<.001$. In (Sudzina, 2016), there was conscientiousness instead of openness to experience in such model, $\mathrm{R}^{2}=.230, \mathrm{R}_{\text {adj }}^{2}=.219$, p-value $<.001$. 


\begin{tabular}{l|l|l|l|l}
\hline Parameter & \multicolumn{1}{|c|}{$\mathrm{B}$} & \multicolumn{1}{|c|}{ Std. Error } & $\mathrm{t}$ & \multicolumn{1}{c}{ Sig. } \\
\hline Intercept & 2.021 & .626 & 3.228 & .002 \\
\hline Mistrust & -.249 & .081 & -3.071 & .002 \\
\hline Agreeableness & .265 & .114 & 2.328 & .021 \\
\hline Conscientiousness & .179 & .103 & 1.737 & .084 \\
\hline Neuroticism & .173 & .094 & 1.837 & .068 \\
\hline
\end{tabular}

Table 7 Parameter estimates of impact of mistrust and selected personality traits on trust

The correlation coefficient between trust and predicted trust from mistrust, agreeableness, conscientiousness, and neuroticism is .339. This would mean Cronbach's alpha of .342. In (Sudzina, 2016), the correlation coefficient was .480 leading to Cronbach's alpha of .545.

If conscientiousness and neuroticism and omitted, i.e. all p-values drop below .05, the correlation coefficient marginally decreases to .293 and related Cronbach's alpha to .273 . In (Sudzina, 2016), a model with all p-values below .05 had a correlation coefficient of .469 and related Cronbach's alpha was .530 .

These values are not good enough for establishing a single-dimension trust indicator because Cronbach's alphas are well below Nunnally's (1978) threshold of .7.

A similar approach could be used to estimate mistrust. But since it is influenced by personality traits less than trust, the correlation coefficient between mistrust and predicted mistrust would be lower than between trust a predicted trust. So, it would not be suitable for a singledimension indicator either.

\section{Conclusions}

The article investigated impact of gender and personality traits on trust, namely on two measures of trust stemming from the standard trust question used for the last 60 years. These two measures of trust (trust and mistrust), though correlated, are not fully opposite to each other, as it was confirmed also here (the correlation coefficient estimated from data at hand is -.226 , not -1 ).

Trust is significantly influenced by agreeableness. The impact of neuroticism on mistrust is borderline significant. Remaining personality traits and gender were not found to be significant. Future research could use a longer version of the questionnaire for the Big Five Inventory or another personality traits framework.

The second goal of the article was to investigate if it is possible to account for gender and personality traits and to create a robust single-dimension trust indicator from two measures of trust. Although personality traits can increase correlation between the two measures of trust, this improvement is not enough for creating a single-dimension indicator. 


\section{References}

Almond, G. and Verba, S., 1963. The Civic Culture. Princeton: Princeton University Press.

Calvo Porral, C. and Levy-Mangin, J. P., 2016. Food private label brands: the role of consumer trust on loyalty and purchase intention. British Food Journal, 118(3), 679-696.

Delina, R. and Drab, R., 2010. Socio-Economic Aspects of Trust Building for the Electronic Business Platforms. E\&M Ekonomie a Management. 13(4), 110-122.

Krasman, J., 2014. Do my staff trust me? The influence of organizational structure on subordinate perceptions of supervisor trustworthiness. Leadership \& Organization Development Journal, 35(5), $470-488$.

Miller, A. and Mitamura, T., 2003. Are Surveys on Trust Untrustworthy? Social Psychology Quarterly, 66(1), 62-70.

Nunnally, J. C., 1978. Psychometric theory, $2^{\text {nd }}$ edition. New York, NY: McGraw-Hill.

Ostroy, J. M. and Starr, R., 1990. The Transaction Role of Money. In Friedman, B., Hahn, F. H. (eds.) Handbook of Monetary Economics. Amsterdam: North-Holland, vol. 1, 3-62.

Peralta, C. F. and Saldanha, M. F. 2014. Knowledge-centered culture and knowledge sharing: the moderator role of trust propensity. Journal of Knowledge Management, 18(3), 538-550.

Rammstedt, B. and John, O. P., 2007. Measuring Personality in One Minute or Less: A 10-Item Short Version of the Big Five Inventory in English and German. Journal of Research in Personality, 41(1), 203-212.

Rosenberg, M., 1956. Misanthropy and Political Ideology. American Sociological Review, 21(6), 690-695.

Smith, T. W., 1997. Factors Relating to Misanthropy in Contemporary American Society. Social Science Research, 26(2), 170-196.

Sudzina, F., 2016. Do gender and personality traits (BFI-10) influence trust? In: Proceedings of the International Scientific Conference of Business Economics Management and Marketing (ISCOBEMM) 2016. Brno: Masaryk University, 31-37.

Wuthnow, R., 1998. Loose Connections: Joining Together in America's Fragmented Communities. Cambridge: Harvard University Press.

František Sudzina

sudzina@business.aau.dk

Aalborg University Faculty of Social Sciences,

Department of Business and Management

2450 København SV, Denmark 